Adalgisa Peixoto Ribeiro ${ }^{a}$

(ID) https://orcid.org/0000-0001-9415-8068

Graziella Lage Oliveira ${ }^{\mathrm{a}}$

(iD) https://orcid.org/0000-0002-3387-3583

Luiz Sergio Silva ${ }^{a}$

(iD) https://orcid.org/0000-0002-3517-6941

Edinilsa Ramos de Souza ${ }^{b}$

(iD) http://orcid.org/0000-0003-0903-4525

a Universidade Federal de Minas Gerais (UFMG), Faculdade de Medicina, Departamento de Medicina Preventiva e Social. Belo Horizonte, MG, Brasil.

b Fundação Oswaldo Cruz (Fiocruz), Escola Nacional de Saúde Pública, Departamento de Estudos sobre Violência e Saúde Jorge Careli.

Rio de Janeiro, RJ, Brasil.

Contato:

Adalgisa Peixoto Ribeiro

E-mail:

adalpeixoto@ufmg.br

Os autores declaram que o trabalho não foi subvencionado e que não há conflitos de interesses.

Os autores informam que o trabalho não foi apresentado em evento científico e que não foi baseado em dissertação ou tese.

\section{Saúde e segurança de profissionais de saúde no atendimento a pacientes no contexto da pandemia de Covid-19: revisão de literatura}

\author{
Occupational safety and health of health workers caring for \\ patients during the Covid-19 pandemic: a literature review
}

\section{Resumo}

Objetivo: analisar a produção científica sobre a saúde dos trabalhadores da Saúde que atendem pacientes no contexto da pandemia de COVID-19. Métodos: realizou-se revisão de literatura que incluiu artigos publicados em 2020, indexados nas bases PubMed, Web of Science e na Biblioteca Virtual em Saúde (Medline e Lilacs). Resultados: foram analisados 52 artigos, segundo grupo profissional estudado, país onde a pesquisa foi realizada, tipo de estudo e tema abordado. Os conhecimentos e questões mais atuais e relevantes e as lacunas existentes sobre o tema foram evidenciados e discutidos do ponto de vista da Saúde Coletiva, particularmente da Saúde do Trabalhador. Mais da metade dos documentos foi produzida na China $(55,7 \%)$ e focalizou as diferentes categorias profissionais de saúde em conjunto $(57,7 \%)$. Estudos do tipo ensaio/opinião $(46,1 \%)$ e estudos transversais $(30,8 \%)$ foram os mais frequentes. Os temas destacados foram o conhecimento dos profissionais sobre a doença, os casos de COVID-19 entre os profissionais, a saúde mental dos trabalhadores da saúde e a segurança no trabalho. Conclusão: os estudos publicados no início da pandemia destacam a insuficiência de conhecimentos atualizados e falhas na proteção da saúde dos trabalhadores e recomendam o gerenciamento dos processos e locais de trabalho, dos casos de COVID-19, das políticas públicas e dos direitos dos trabalhadores.

Palavras-chave: profissionais de saúde; COVID-19; saúde do trabalhador.

\begin{abstract}
Objective: to analyze the academic production on the occupational health of health workers serving patients during the COVID-19 pandemic. Methods: a literature review including articles from 2020 indexed in PubMed, Web of Science and Biblioteca Virtual em Saúde (Medline and Lilacs). Results: we analyzed 52 articles according to professional group studied, country were the research was conducted, type of study design and research theme. We emphasized and discussed the most updated and relevant knowledge, questions and issues from the point of view of Collective Health, and Worker's Health. Over half of the papers were from China (55.7\%) and studied different health professionals categories grouped (57.7\%). The most frequent studies types were essays (46.1\%) and cross-sectional studies (30.8\%). The highlighted themes were the workers' knowledge about the disease, Covid-19 cases among health workers, mental health, and occupational safety. Conclusion: the studies published at the beginning of the pandemic highlight lack of updated knowledge and failures in protecting workers' health, and recommend the management of work processes, workplaces, COVID-19 cases, public policies and workers' rights.
\end{abstract}

Keywords: health workers; COVID-19; occupational health. 


\section{Introdução}

Em dezembro de 2019, a Organização Mundial da Saúde (OMS) divulgou que as autoridades chinesas tinham detectado um novo vírus identificado em uma pessoa hospitalizada com pneumonia em Wuhan, posteriormente denominado SARS-CoV-2. Cinco meses após, o número de casos atingiu milhões de pessoas e o ataque viral ceifou milhares de vidas. O mundo vivencia uma pandemia sem precedentes na história contemporânea ${ }^{1}$.

A pandemia de COVID-19, como é chamada a doença provocada pelo SARS-CoV-2, atinge os serviços de saúde impondo uma demanda extra de estruturas, insumos e recursos humanos, o que tem desafiado de maneira diferenciada os sistemas nacionais de saúde dos diversos países. O Brasil, onde historicamente o financiamento é insuficiente para garantir as condições para gerir as demandas cotidianas, está enfrentando sérias adversidades com o advento da pandemia de COVID-19. No fechamento deste texto, o país somava mais de 1,1 milhão de casos confirmados da doença e 53.800 mortes $^{2}$.

Os profissionais de saúde, grupo composto por distintas categorias profissionais, estão diretamente implicados no atendimento às pessoas infectadas pela COVID-19 e, por este motivo, compõem um grupo de risco específico para a infecção. A pandemia em curso expôs a fragilidade do setor de Saúde em garantir a segurança dos profissionais envolvidos no cuidado aos infectados. Trata-se de uma exposição que pode ser compreendida como "exposição biológica" e a maioria, se não todos os profissionais de saúde, estão expostos e possuem alto risco de adquirir a doença, particularmente ao realizar procedimentos em vias aéreas ou próximos a elas ${ }^{3,4}$.

Este, sem dúvida, é um dos grandes desafios dessa pandemia: garantir a segurança e a proteção efetiva dos trabalhadores da saúde em um cenário de muitas dúvidas ${ }^{5}$ e poucas certezas, considerando-se o insuficiente conhecimento sobre as formas de tratar e controlar a doença e, principalmente, a sua alta transmissibilidade e velocidade de disseminação $0^{6}$.

Com a finalidade de ampliar o conhecimento disponível sobre a doença e compreender sua dinâmica de transmissão entre os profissionais de saúde expostos à COVID-19, várias instituições como a OMS, o Centers for Diseases Control and Prevention (CDC) e outras instituições e entidades têm disponibilizado diariamente informações, protocolos e recomendações atualizadas ${ }^{7,8}$.
Diversos estudos nacionais e internacionais têm sido realizados para investigar a incidência e as repercussões da COVID-19 nos profissionais de saúde, bem como suas formas de transmissão. Organizar e sistematizar este conhecimento é fundamental para fornecer orientações aos gestores e demais tomadores de decisão no sentido de minimizar os riscos, fornecer equipamentos e insumos de proteção e reduzir os casos de afastamento de profissionais por contágio e/ou infecção por COVID-19 ${ }^{9}$.

O objetivo deste artigo é identificar e analisar a produção científica nacional e internacional acerca da saúde e segurança ocupacional dos trabalhadores da saúde durante a pandemia de COVID-19.

\section{Métodos}

Trata-se de uma revisão de literatura nas bases bibliográficas PubMed, Web of Science e Biblioteca Virtual em Saúde (MEDLINE e LILACS). A estratégia de busca incluiu os termos "health personnel", "occupational health", "medical staff", "health care personnel", juntos com "coronavirus" e "COVID19”. Foram incluídas publicações realizadas em 2020, sem limite de idiomas, considerando somente artigos completos. A busca foi realizada entre os dias 18 e 28 de abril de 2020 e localizou 322 documentos, dos quais 78 foram excluídos por não terem resumo (editoriais, cartas ao editor, documentos governamentais, entre outros), e 33 por se repetirem em mais de uma base. A seleção seguiu com 211 artigos que tiveram seus títulos e resumos lidos. Nos casos em que essa leitura não permitiu que se decidisse sobre a inclusão do texto, procedeu-se à leitura do artigo na íntegra. Essa fase do processo foi realizada por dois pesquisadores independentes e, posteriormente, as dúvidas e discrepâncias identificadas foram discutidas entre os autores deste artigo para tomarem a decisão final.

Os textos selecionados nesta última etapa foram analisados da seguinte forma: 1) caracterização do grupo focalizado segundo categoria profissional (equipe de saúde, quando as categorias profissionais dos trabalhadores não eram diferenciadas; médicos e algumas especializações médicas, e outras categorias da área da saúde como enfermeiros, odontólogos, farmacêuticos e outros); o país de origem/foco da pesquisa e o tipo de estudo realizado (caso-controle, transversal, revisão, relato de caso/série de casos e ensaio/opinião); e 2) classificação dos temas a partir da leitura dos resumos e identificação de conteúdos clássicos da área da Saúde do Trabalhador e/ou por similaridade de objetos de estudo (conhecimentos 
sobre a COVID-19 e atitudes de profissionais de saúde; casos de COVID-19 na equipe de trabalhadores da saúde; saúde mental de profissionais de saúde em tempos de pandemia; e segurança ocupacional). Os temas foram definidos de forma consensual pelos autores e agregados a partir da literatura do campo teórico/conceitual da Saúde do Trabalhador.

Os dados são apresentados em tabela com frequências absolutas e relativas. Além disso, os temas focalizados nos estudos são detalhados e posteriormente discutidos em diálogo com a literatura para identificação de avanços e lacunas do conhecimento agregado pelo acervo analisado.

\section{Resultados}

Ao final das etapas de busca e seleção foram analisados 52 documentos, conforme pode ser visto na Figura 1.
O acervo analisado focalizou principalmente equipes de trabalhadores que atuam em hospitais, como médicos e enfermeiros, citados como "equipes de saúde" ou "equipes médicas", e também se dirigiu a algumas categorias médicas de forma específica, como cirurgiões, obstetras, urologistas, oftalmologistas, anestesistas e psiquiatras; e outros profissionais de saúde como radiologistas, farmacêuticos e odontólogos. Estudantes da área da saúde e profissionais administrativos também foram considerados. A China produziu $55,7 \%$ dos textos, seguida por Estados Unidos da América (EUA) com 11,5\%. A distribuição dos tipos de estudos analisados revelou que os ensaios/opinião foram os mais frequentes, seguidos pelos estudos transversais (Tabela 1). No conjunto dos estudos foi incluído um total de 20.986 trabalhadores de saúde, sendo 98,9\% deles em estudos do tipo transversal, $0,6 \%$ em estudos caso controle e $0,5 \%$ nos relatos de caso.

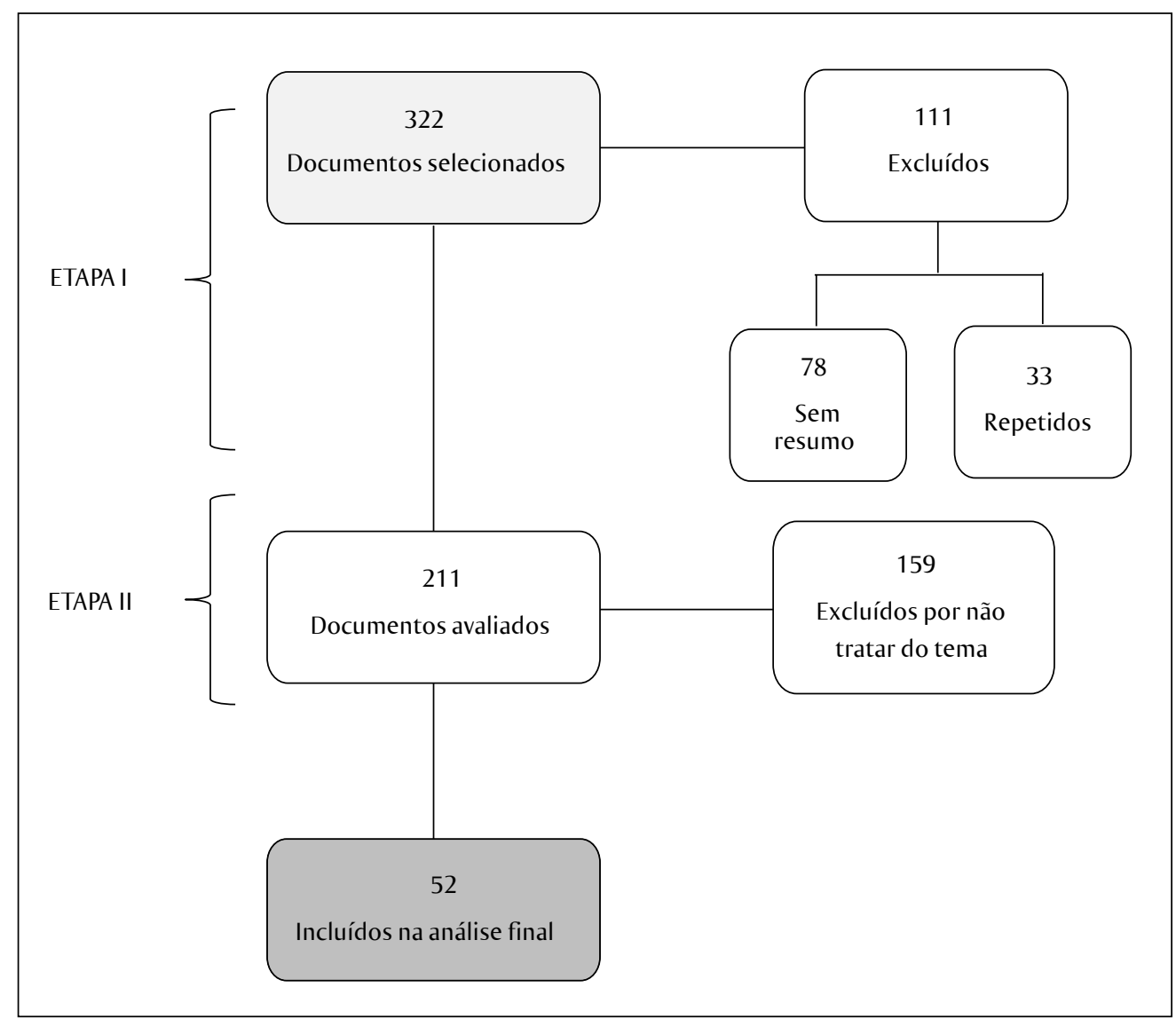

Figura 1 Fluxograma das etapas do processo de seleção dos documentos 
Tabela 1 Distribuição das publicações segundo grupo focalizado, tipo de estudo, país de origem e temas abordados

\begin{tabular}{|c|c|c|c|}
\hline Variáveis & Referência & $N$ & $\%$ \\
\hline \multicolumn{4}{|l|}{ Grupo Analisado* } \\
\hline Profissionais de saúde (em geral) & $\begin{array}{l}3,9,10,11,14,16,17,18,19,20,21,23,24,25 \\
26,27,29,32,34,35,36,37,38,39,46,47,49 \\
57,58,59,60\end{array}$ & 31 & 59,6 \\
\hline Médicos (em geral) & $22,28,30,31,41,61$ & 6 & 11,5 \\
\hline Cirurgiões & 33,42 & 2 & 3,8 \\
\hline Enfermeiros & $12,22,28,30,31,50,61,62$ & 8 & 15,4 \\
\hline Odontologistas & $13,48,63$ & 3 & 5,8 \\
\hline Estudantes & $10,27,64$ & 3 & 5,8 \\
\hline Outras categorias*** & $11,12,15,26,40,43,44,45,65$ & 9 & 17,3 \\
\hline \multicolumn{4}{|l|}{ Tipo de estudo } \\
\hline Caso-controle & 18 & 1 & 1,9 \\
\hline Transversal & $\begin{array}{l}10,11,12,13,20,22,24,25,26,27,28,29,30 \\
31,38,62\end{array}$ & 16 & 30,8 \\
\hline Revisão & $14,15,17,37,40,41,59,65$ & 8 & 15,4 \\
\hline Relato de caso/série de casos & $21,23,57$ & 3 & 5,8 \\
\hline Ensaio/opinião & $\begin{array}{l}3,9,16,19,32,33,34,35,36,39,42,43,44,45 \\
46,47,48,49,50,58,60,61,63,64\end{array}$ & 24 & 46,1 \\
\hline \multicolumn{4}{|l|}{ Origem/país } \\
\hline China & $\begin{array}{l}12,17,21,22,23,25,26,27,28,29,30,31,32 \\
34,38,39,42,43,44,45,47,48,49,50,57,62 \\
63,64,65\end{array}$ & 29 & 55,7 \\
\hline EUA & $18,40,41,58,59,61$ & 6 & 11,5 \\
\hline Brasil & $16,19,35$ & 3 & 5,8 \\
\hline Itália & $3,9,11$ & 3 & 5,8 \\
\hline Outros & $10,13,20,24,36,37,46,60$ & 8 & 15,4 \\
\hline Não identificado & $14,15,33$ & 3 & 5,8 \\
\hline \multicolumn{4}{|l|}{ Temas abordados } \\
\hline $\begin{array}{l}\text { Conhecimentos sobre COVID-19 e atitudes de } \\
\text { profissionais de saúde }\end{array}$ & $9,10,11,12,13,14,16,61,64$ & 9 & 17,3 \\
\hline Casos de COVID-19 na equipe & $17,18,20,21,22,23,24,57$ & 8 & 15,4 \\
\hline $\begin{array}{l}\text { Saúde mental de profissionais de saúde em tempos } \\
\text { de pandemia }\end{array}$ & $25,26,27,28,29,30,31,62$ & 8 & 15,4 \\
\hline Segurança ocupacional & $\begin{array}{l}3,15,19,32,33,34,35,36,37,38,39,40,41,42 \\
43,44,45,46,47,48,49,50,58,59,60,63,65\end{array}$ & 27 & 51,9 \\
\hline
\end{tabular}

* Total não soma $100 \%$, pois um único artigo pode abordar mais de um grupo de profissionais.

*** Outras categorias: inclui obstetras, oftalmologistas, anestesiologistas, urologistas, radiologistas, psiquiatras, farmacêuticos, profissionais administrativos.

****:**: Outros: inclui Reino Unido, Turquia, Índia (2), Jordânia, Indonésia, Holanda, Austrália.

Mais da metade dos artigos (51,9\%) se dedicou ao tema da "Segurança ocupacional", seguido pela preocupação com a "Saúde Mental dos profissionais de saúde frente à pandemia" e pelos demais temas (Tabela 1).

A seguir, são detalhados os principais conteúdos dos textos, segundo os quatro temas de análise: conhecimentos sobre a COVID-19 e atitudes de profissionais de saúde; casos de COVID-19 na equipe de trabalhadores da saúde; saúde mental de profissionais de saúde em tempos de pandemia; e segurança ocupacional.

\section{Conhecimentos sobre a COVID-19 e atitudes de profissionais de saúde}

No conjunto dos nove textos $(17,3 \%$ dos 52 analisados) que abordam conhecimentos e práticas adotadas pelas equipes de saúde em relação à COVID-19, observou-se que a consciência geral sobre a doença, 
que inclui conhecimento sobre sua transmissão, conceito de "contato próximo", apresentações clínicas, práticas de controle de infecção, gerenciamento e tratamento de casos da doença, entre outros, foi considerada adequada entre médicos, enfermeiros e estudantes de medicina ${ }^{10,11}$, atuantes em hospitais gerais de ensino e que atendem pacientes com a doença. Resultado semelhante foi encontrado em outro estudo que analisou especificamente os psiquiatras que atuam em hospitais psiquiátricos ${ }^{12}$. A maioria dos odontólogos está ciente dos sintomas, modo de transmissão e controle da COVID-19, mas apresentaram uma compreensão limitada sobre como proteger sua equipe e os pacientes ${ }^{13}$. Por outro lado, a categoria de funcionários administrativos foi a que apresentou menor conhecimento, demonstrando a necessidade de intervenções educacionais e de treinamento para estes profissionais, abordando práticas de controle de infecção para a COVID-1910.

Entre os profissionais que atuam em serviços não diretamente ligados ao atendimento de urgência para COVID-19, como serviços de saúde mental, um dos estudos analisados, realizado na China com 311 profissionais de psiquiatria (médicos e enfermeiros), identificou que $77,2 \%$ deles expressaram desejo de cuidar de pacientes psiquiátricos com a doença ${ }^{12}$. No entanto, destacou que ainda falta treinamento para a assistência em saúde mental de doentes com COVID-19 e que o sentimento de medo é uma realidade entre essas equipes de saúde ${ }^{14}$. Soma-se a isto o fato de que alguns profissionais de outras áreas, como urologistas, por exemplo, estão sendo deslocados de suas atividades usuais para atuar no cuidado a pacientes com COVID-19. Nesse caso, o estudo indica que os profissionais sejam orientados adequadamente sobre diagnóstico e manejo de pacientes com a doença e as adaptações necessárias em sua prática profissional ${ }^{15}$.

Spinazzè et al. ${ }^{9}$ destacaram a necessidade de conhecimento profissional no campo da Higiene Ocupacional para enfrentar o contexto de emergência, que exige a tomada de decisões baseadas em evidências fornecidas pela literatura científica disponível, por organismos nacionais e internacionais e pela observância das disposições legais. Nesse sentido, destaca a importância da circulação de orientações operacionais para todas as categorias envolvidas no serviço de saúde, com o objetivo de traduzir indicações gerais em procedimentos operacionais claros, a serem adotados nos locais de trabalho.

Correia et al. ${ }^{16}$ acrescentam que, além dos desafios enfrentados para conhecer as características da doença, o "dilúvio de informações" também é prejudicial. Relatam o alto número de publicações científicas, abrangendo as mais diversas metodologias (estudos in vitro, estudos de caso, estudos clínicos observacionais, randomizados e revisões sistemáticas), que aumentam a insegurança quando se considera que uma parcela dos profissionais de saúde não possui conhecimento adequado para avaliar criticamente o método científico utilizado.

O desconhecimento sobre a doença tem sido apontado como uma das causas do aumento de contágio, inclusive entre os profissionais da saúde ${ }^{17}$.

\section{Casos de COVID-19 na equipe de trabalhadores da saúde}

Oito artigos $(15,4 \%)$ do acervo analisado se dedicaram a identificar as taxas de incidência e prevalência da doença entre os profissionais de saúde, bem como sua caracterização epidemiológica. Heinzerling et al. ${ }^{18}$ relatam a exposição não protegida como causa dos primeiros casos documentados de contaminação profissional nos EUA. De acordo com estudo realizado por Kowalski et al. ${ }^{19}$, os profissionais de saúde representam de $3,8 \%$ a $20 \%$ da população infectada no Brasil, mostrando o grande impacto da doença na força de trabalho. Ao testar 1.533 trabalhadores da saúde com sintomas sugestivos de COVID-19, no Reino Unido, Keeley et al. ${ }^{20}$ identificaram que $18 \%$ foram positivos.

Estudo de Chu et al. ${ }^{21}$ demonstrou que em um hospital chinês foram hospitalizados 54 profissionais da equipe médica, infectados pelo SARS-Cov-2, entre 7 de janeiro e 11 de fevereiro de 2020. A maioria deles era de outros departamentos clínicos $(72,2 \%)$, e não do serviço de emergência (3,7\%), ou departamentos de tecnologia médica $(18,5 \%)$. Liu et al. ${ }^{22}$ analisaram 30 casos de COVID-19 (22 médicos e 8 enfermeiros) e identificaram que os profissionais que atuaram em hospitais apresentaram maior risco de infecção; aqueles que apresentaram manifestações mais graves tiveram tempo de febre prolongado, contagem de glóbulos brancos, de linfócitos, dímero D e nível de albumina alterados e lesões no fígado e miocárdio.

Xing et al. ${ }^{23}$ acompanharam 62 médicos atuantes em hospital de Wuhan, China, diagnosticados com COVID-19 por RT-PCR e utilizaram os resultados para estabelecer critérios de liberação após a recuperação. Observaram que mesmo depois de recuperados os pacientes ainda podiam carregar uma pequena carga viral de difícil detecção, que, no entanto, não os caracterizava como transmissores da doença. Relataram a possibilidade de recaída, com piora do quadro em alguns casos.

Estudo holandês realizado por Reusken et al. ${ }^{24}$ testou 1.097 trabalhadores de saúde que atuavam em hospitais e tinham queixas respiratórias leves e identificou que $4,5 \%$ deles testaram positivo para COVID-19. As taxas de infecção entre esses profissionais, com sintomas respiratórios moderados ou sem 
dados epidemiológicos positivos, foram usadas como uma aproximação das taxas de transmissão comunitária na Holanda.

\section{Saúde mental de profissionais de saúde em tempos de pandemia}

Oito artigos $(15,4 \%$ dos 52 analisados) trataram do tema saúde mental, realizando estudos do tipo transversal envolvendo um total de 9.514 profissionais de saúde. Alguns desses estudos demonstraram que os profissionais que estavam no atendimento imediato, que incluía diagnóstico e tratamento de pacientes com COVID-19, experimentaram maior impacto em sua saúde mental.

Trabalhar na linha de frente se mostrou um fator de risco para os sintomas de ansiedade, depressão, insônia, angústia e medo ${ }^{25-27}$. A prevalência de ansiedade variou de $23 \%$ a $44,6 \%$ na equipe médica (médicos e enfermeiros) ${ }^{25,26,28}$. Sintomas depressivos foram identificados em 50,4\% dos 1.257 profissionais estudados por Lai et al. ${ }^{25}$ na China. Ser profissional de enfermagem do sexo feminino esteve associado a sintomas graves de depressão, angústia ${ }^{25}$ e ansiedade ${ }^{25,28}$. Ao comparar os sintomas de estresse psicológico entre equipe médica e estudantes de medicina, Wu et al. ${ }^{27}$ identificaram que os profissionais são mais susceptíveis a essa condição. Xiao et al. ${ }^{29}$ observaram que os níveis de apoio social com que as médicas podiam contar estavam positivamente associados à autoeficácia e qualidade do sono e negativamente associados ao grau de ansiedade e estresse.

Cai et al. ${ }^{30}$ estudaram profissionais da equipe médica atuando na linha de frente dos cuidados a pacientes de COVID-19 na província de Hubei, China, identificando que a disponibilidade de diretrizes rígidas de controle de infecção, de equipamentos especializados, de reconhecimento profissional por parte dos gestores hospitalares e governamentais e a concreta redução dos casos da doença, proporcionariam benefícios psicológicos a toda a equipe.

A Síndrome de Burnout, condição de esgotamento físico e mental associada à vida profissional, mostrou-se menos prevalente entre os que atuam na linha de frente do atendimento a pacientes de COVID-19 se comparados aos profissionais que trabalham em outras enfermarias (13\% versus $39 \%$, respectivamente) ${ }^{31}$. Segundo os autores, este resultado inesperado pode indicar que os primeiros, ao abordarem diretamente o COVID-19, puderam ter a percepção de maior controle da situação e do local de trabalho, o que favoreceu seu engajamento evitando o desgaste. Além disso, puderam estar mais próximos dos principais tomadores de decisão e com acesso a informações mais oportunas e precisas ${ }^{31}$.

\section{Segurança ocupacional}

Entre os estudos analisados, 51,9\% buscaram compreender ou avaliar as condições de saúde e segurança dos trabalhadores da área da saúde durante a pandemia. Grande parte desses artigos (75\%) abordou a utilização de Equipamentos de Proteção Individual (EPI) como uma das medidas consideradas mais relevantes para evitar a contaminação por COVID-19 durante o atendimento de pacientes suspeitos ou confirmados da doença.

Os EPI foram descritos conforme os níveis de exposição, e classificados em: nível I - roupas de trabalho, capotes, máscaras cirúrgicas; nível II - todos listados no nível I, com substituição da máscara cirúrgica pela N95, e acrescidos do uso de óculos, roupas protetoras, roupas impermeáveis e protetores de calçados; e nível III - todos listados no nível II, acrescidos de protetores faciais e salas com uso de pressão positiva ${ }^{26,32-34}$.

Contudo, para que os EPI sejam realmente eficazes, é fundamental que os profissionais de saúde sejam previamente treinados quanto à paramentação e à desparamentação, bem como informados quanto às medidas de propagação e mitigação da doença ${ }^{35}$. Os estudos mostraram que a utilização de EPI pelos profissionais de saúde reduz os riscos de infecção por COVID-19 e leva a um aumento na sensação de segurança dos que atuam em hospitais ${ }^{36,37}$. Jiang et al. ${ }^{38}$ descreveram uma prevalência de $43 \%$ de lesões de pele causadas pelos EPI, cujos fatores responsáveis foram a pressão local, a sudorese intensa pelo calor e o longo tempo de uso, principalmente quando no caso dos EPI de nível III.

Entre as orientações relacionadas aos EPI, destacam-se medidas preventivas mais abrangentes, como a melhoria da qualidade e da ergonomia desses equipamentos e a redução do tempo de uso associado a medidas de proteção coletiva ${ }^{39}$. No acervo analisado, orientações sobre EPI e segurança dos profissionais são detalhadas considerando procedimentos em otorrinolaringologia $^{19,34}$, obstetrícia ${ }^{40}$, ortopedia ${ }^{41}$, cirurgia bucomaxilofacial ${ }^{32}$, neurocirurgia ${ }^{42}$, cirurgia robótica ${ }^{33}$, cirurgia colorretal ${ }^{39}$, oftalmologia ${ }^{43}$, anestesia $^{44}$, radiologia ${ }^{45}$ e urologia ${ }^{15}$.

Os artigos ressaltam que a utilização de EPI pelos profissionais de saúde por si só não é suficiente para interromper a propagação da doença. São necessárias medidas adicionais que considerem outros fatores do ambiente de cuidado, por exemplo: triar os pacientes que se apresentam para atendimento (identificação do caso); colocar máscara e isolar rapidamente os doentes; reduzir o número de pessoas na sala de espera e de atendimento (distanciamento físico); avaliar o risco dos procedimentos a serem realizados; 
higienizar as mãos, limpar e desinfetar os ambientes de trabalho; gerenciar os resíduos hospitalares; usar tecnologias emergentes, como telemedicina hospitalar e aplicativos de compartilhamento de arquivos on-line, que podem facilitar o acompanhamento de pacientes nas consultas eletivas ou para outros problemas de saúde com menor gravidade ${ }^{32,36,40,43,46-48}$.

Nas cirurgias de urgência e emergência, são recomendadas condutas como traçar planos objetivos e estabelecer critérios de classificação de risco de infecção, considerando a história médica, os sintomas clínicos, os exames laboratoriais dos pacientes e a adoção de protocolos de segurança ${ }^{16,44,49}$ para selecionar materiais e/ou técnicas de proteção mais adequadas a cada caso, podendo ser adaptadas de acordo com a realidade de cada serviço ${ }^{3,9,16,44,49}$. Em procedimentos respiratórios, é recomendável garantir o uso da sedação adequada para reduzir a emissão de gotículas e reduzir o número de profissionais expostos durante os procedimentos ${ }^{3}$.

Além dessas medidas, foi destacada a necessidade de elaborar políticas direcionadas ao cuidado com os profissionais expostos a longas e exaustivas jornadas, como a criação de escalas de turnos coerentes para a atuação dos profissionais da saúde, permitindo descanso e realização de necessidades fisiológicas como alimentação e uso de sanitários ${ }^{50}$, além do afastamento dos profissionais classificados como grupo de maior vulnerabilidade à infecção e das atividades de risco de contaminação ${ }^{35}$.

\section{Discussão}

Os resultados deste estudo demonstram que os profissionais de saúde que atuam em hospitais e na linha de frente das ações de enfrentamento ao COVID-19 foram os mais focalizados na produção científica sobre trabalhadores da saúde, publicada no início da pandemia (janeiro a abril de 2020). $\mathrm{Na}$ maioria dos artigos, as equipes de saúde, formadas essencialmente por médicos e enfermeiros, estiveram no centro das preocupações dos pesquisadores, pelo alto risco de contaminação no contato direto com os pacientes. Além disso, trata-se de uma doença que ainda não é completamente conhecida pela comunidade científica, com exigências cada vez mais específicas em termos de procedimentos, medicamentos e insumos para seu tratamento e controle.

No conjunto de artigos analisados foram observados, em sua maioria, estudos transversais ou relatos de experiência. Isso provavelmente decorre do ineditismo e da velocidade de propagação da doença pelo mundo, impossibilitando a realização de estudos epidemiológicos que demandem seguimento, motivo pelo qual são mais demorados. Visto que a ciência se constitui como grande fonte geradora de informações confiáveis, muitos pesquisadores e periódicos científicos iniciaram um processo de divulgação rápida de estudos sobre a COVID-19. Certamente ensaios clínicos envolvendo vacinas, estudos de caso-controle e de coorte com profissionais de saúde estão sendo conduzidos e serão publicados oportunamente.

A China, por exemplo, país onde a epidemia de COVID-19 teve início, acumula, até a data deste levantamento, a mais expressiva publicação de estudos que contemplam os profissionais de saúde atuando na atenção aos pacientes no contexto da pandemia. No entanto, é importante considerar que essa produção de conhecimento tem se ampliado à medida que o SARS-CoV-2 avança e atinge, de forma diferenciada, os países e suas populações. Um percentual de $11,5 \%$ dos estudos foi realizado nos EUA, que atualmente é o país com o maior número de casos da doença, seguido pelo Brasil, em segundo lugar no número de pessoas infectadas e de mortes ${ }^{51}$. O Brasil contribuiu com número menor de estudos (5,8\%), mas é bom registrar que a produção nacional e internacional sobre o tema está em expansão.

A fim de preparar as equipes de saúde para enfrentar o avanço da infecção e a gravidade com que se manifesta em alguns casos, capacitações e treinamentos foram elaborados em tempo recorde. Isso foi necessário porque os conhecimentos básicos sobre o modo de transmissão e as medidas de controle de doenças infecciosas em geral não foram suficientes para frear a disseminação da doença, particularmente entre profissionais de saúde. A COVID-19 tem desafiado os conhecimentos prévios de diversas áreas da ciência e da medicina ${ }^{52}$.

Desde os primeiros casos diagnosticados em profissionais da saúde em Wuhan, na China, tenta-se compilar e divulgar o que já se sabe sobre como se proteger do risco de contaminação biológica. A falta de conhecimento sobre os mecanismos de disseminação (o papel dos assintomáticos, por exemplo), contágio e manifestações clínicas, tem colocado em risco algumas categorias de profissionais que atuam na área da saúde, como pessoal administrativo e de tecnologia de diagnóstico, por exemplo os profissionais de radiologia. Tal desconhecimento sobre a doença, embora em redução, tem sido apontado como uma das causas do aumento de contágio entre os profissionais da saúde ${ }^{17}$.

É interessante destacar que os próprios profissionais de saúde estão sendo desafiados a buscar conhecimento ao mesmo tempo em que atendem aos casos de COVID-19 que sobrecarregam os serviços, realizam o acompanhamento de pacientes com outros problemas de saúde, principalmente doenças 
crônicas, e se protegem da exposição ao risco de adoecer. Muitos pacientes que buscam atendimento, mesmo infectados, podem permanecer assintomáticos e, sem o diagnóstico da doença, podem aumentar o risco de contaminação dos profissionais de saúde. Heinzerling et al. ${ }^{18}$ relatam a exposição não protegida como causa dos primeiros casos documentados de contaminação profissional nos EUA.

Pesquisadores da área de Saúde do Trabalhador discutem que o exercício das atividades laborais e as condições de trabalho podem ser fontes potenciais de exposição ao vírus e que as recomendações já sistematizadas incluem medidas individuais de biossegurança e proteção, que são essenciais, mas insuficientes ${ }^{53}$. As condições de trabalho a que esses profissionais estão expostos e as políticas de organização e estruturação do trabalho são fundamentais nessa análise sobre saúde e segurança. No Brasil, acumulam-se relatos sobre a precarização dessas condições, inadequações de higiene e de EPI, jornadas de trabalho ampliadas e exaustivas e falta de treinamento.

Conhecer o número de casos de COVID-19 entre trabalhadores de saúde é importante para que se tomem as decisões em saúde pública e, particularmente, em saúde do trabalhador. Alguns artigos analisados apresentam importantes contribuições para o mapeamento de condições de trabalho que auxiliam a formular indicadores de maior risco, tais como faixa etária, exposição sem proteção adequada e área de atuação (os que estão no atendimento clínico se contaminam mais do que os de tratamento intensivo e os de urgência/emergência ${ }^{21}$, e os enfermeiros mais do que os médicos ${ }^{50}$ ).

A testagem de espécimes, tanto de oro e nasofaringe quanto sorológicas, configura-se como uma estratégia que possibilita planejar o controle e reduzir a exposição entre profissionais e pacientes. $\mathrm{O}$ conjunto de estudos analisados mostra que a testagem dos profissionais de saúde pode contribuir para otimizar a força de trabalho, subsidiar afastamentos dos que estão contaminados e oferecer segurança aos que testarem negativo ${ }^{20}$. Além dos profissionais de saúde, a testagem dos familiares desses trabalhadores pode auxiliar a reduzir o absenteísmo decorrente do contato ou da necessidade de prestação de cuidados à família. É importante salientar que a doença tem sido apontada como importante causador da redução da força de trabalho entre os profissionais de saúde, com prejuízos para o atendimento à população.

No Brasil, as disparidades inter e intrarregionais e as políticas adotadas para o setor da saúde nos últimos anos fragilizaram o Sistema Único de Saúde (SUS). Com o surgimento da pandemia de COVID-19, as deficiências tornaram-se mais intensas e expostas, atingindo diretamente os $70 \%$ da população que depende dos serviços públicos de saúde e os profissionais que enfrentam condições de trabalho precárias e inadequadas em termos de atuação e de proteção individual. Até o dia 13 de maio de 2020, dados do e-SUS indicavam que 31.790 profissionais de saúde tinham sido contaminados com a doença $^{54}$. O número de profissionais de enfermagem infectados no Brasil soma 21.524, com uma letalidade de $2,29 \%{ }^{55}$.

As incertezas, a sobrecarga de trabalho e os riscos a que os profissionais estão expostos com a crescente demanda de pacientes infectados e que necessitam de cuidados hospitalares de enfermaria e de terapia intensiva afetam também a saúde mental desses trabalhadores. Esses impactos parecem ser especialmente mais intensos para as mulheres, que precisam conciliar sua dedicação e preocupações relativas ao trabalho com sua saúde, com a família e com os afazeres domésticos. O apoio social dispensado a elas pode interferir diretamente em sua qualidade de vida e saúde em geral ${ }^{29}$. O suporte aos profissionais que têm filhos em idade escolar e/ou são do grupo de risco, por faixa etária ou outra condição clínica, é fundamental e pode ser decisivo para a continuidade de sua atividade laboral ${ }^{16}$.

Mesmo com as repercussões negativas destacadas, é interessante mencionar que os profissionais de saúde acreditam que têm responsabilidade social e profissional com o atendimento dos pacientes e com a realização de jornadas estendidas. No entanto, isso se soma às preocupações com sua segurança e a de suas famílias, o que afeta a saúde mental, principalmente por presenciarem as mortes decorrentes da COVID-19. A adoção de diretrizes rígidas de controle de infecção, disponibilização e adequação de equipamentos especializados, aliados à valorização do trabalho, por parte dos gestores hospitalares e governamentais, e a concreta redução dos casos da doença podem proporcionar benefícios à saúde mental de toda a equipe ${ }^{30}$.

Os artigos têm recomendações específicas para os profissionais de saúde, que incluem treinamento das equipes, uso correto dos EPI adequados em quantidade e tipo, indicação de suspensão de procedimentos eletivos para reduzir o risco de exposição dos pacientes a condições vulneráveis ao COVID-19 e medidas para controle da infecção. Além disso, possuem também indicações mais amplas direcionadas à população em geral e aos gestores. Trata-se de advertências sobre a adoção de distanciamento social ampliado, com a função de reduzir a disseminação do vírus e a imposição de bloqueio em larga escala para que seja possível preparar os serviços de saúde para lidar com o aumento da demanda de cuidados ${ }^{46}$. Assim como indicado pelos Conselhos de Medicina, exortam o uso de estratégias de telemedicina (teleorientação, telemonitoramento e teleconsulta) para favorecer o 
acompanhamento de pacientes com condições crônicas e mesmo para as emergências de menor gravidade, protegendo o binômio profissional/paciente ${ }^{3,16}$.

No que tange às orientações sobre a gestão, segurança e ambiente dos serviços de saúde, os trabalhos apontam para a necessidade da formação de equipes exclusivas para atender os pacientes com COVID-19, com estabelecimento de fluxos bem demarcados. Além disso, recomendam realizar análise eficaz de insumos especializados, com atualização constante da estrutura regulatória, desenvolvimento e circulação de orientações operacionais para os mais diversos setores internos (profissionais de saúde, administrativos e pacientes) para traduzir indicações gerais em procedimentos operacionais claros e ferramentas de implementação a serem adotadas nos locais de trabalho ${ }^{9}$.

Destacam-se como lacunas do conhecimento no acervo de trabalhos analisados, a abordagem do treinamento e desenvolvimento de profissionais, que atuavam em outras áreas da saúde e estão sendo deslocados para atendimento a pacientes graves de COVID-19 e seus impactos na saúde geral e mental desses profissionais; o agravamento de transtornos mentais, incluindo as tentativas e os casos de suicídio e as crises de ansiedade e pânico entre os profissionais de saúde; questões como estigma e violência física e psicológica, já identificadas em comunicações da imprensa e em alguns documentos da OMS ${ }^{56}$. Outro ponto importante que se reflete sensivelmente na saúde mental dos profissionais são as pressões e frustrações diante da necessidade de tomada de decisões ante o número de pacientes gravemente doentes e a estrutura disponível para oferecer cuidados. Destaca-se ainda a ausência de estudos qualitativos que deem voz aos profissionais de saúde sobre as experiências vivenciadas em seu fazer laboral em um contexto de extrema tensão, sobrecarga e exigências físicas e cognitivas, que subsidiem políticas de apoio técnico e psicológico.

Finalmente, as recomendações dos artigos são convergentes com o preconizado por organismos internacionais como o Centers for Disease Control and Prevention (CDC), pelo National Institute for Occupational Safety and Health (Niosh), o Occupational Safety and Health Administration (OSHA), e a OMS. Elas podem ser resumidas em: a) Gerenciamento geral - que inclui avaliação dos locais e tarefas realizadas quanto aos níveis de risco e de exposição, organização do distanciamento social nas unidades, monitoramento e divulgação das comunicações oficiais de saúde pública para gestores e trabalhadores; b) Gerenciamento do trabalho - estabelecimento de formas de lidar com o absenteísmo, organização de turnos de trabalho (alternância e formação de equipes para aumentar as medidas de distanciamento e redução do número de trabalhadores nos locais) e do trabalho remoto, adiamento de procedimentos eletivos, fornecimento de EPI conforme o tipo de risco e exposição; c) Gerenciamento dos locais de trabalho - redução do uso de ferramentas de trabalho em comum, limpeza e desinfecção rotineiras de superfícies, equipamentos e outros elementos do ambiente de trabalho; d) Gerenciamento dos casos - pronta identificação e isolamento dos doentes, afastamento de suspeitos, monitoramento de sinais e de testagens específicas adequadas, facilitação do teleatendimento e telediagnóstico, e) Gerenciamento de políticas e direitos dos trabalhadores - estabelecimento de políticas de afastamento para trabalhadores doentes ou do grupo de risco com manutenção de seus direitos trabalhistas durante o período de pandemia.

\section{Conclusão}

Com a emergência sanitária em decorrência da pandemia causada pelo SARS-Cov-2, importantes questões de proteção e cuidados com a saúde dos trabalhadores estão surgindo.

Desde o primeiro caso até o fechamento deste texto, diversos pesquisadores estão publicando suas experiências, principalmente em países que têm vivenciado grande impacto da doença, como a China, a Itália, os Estados Unidos da América e o Reino Unido. Tem sido um desafio contínuo produzir conhecimento cientificamente sólido, ferramentas apropriadas e metodologias eficazes que evoluam à medida que avança o número de casos de COVID-19 no mundo e o conhecimento sobre o comportamento da doença e as experiências de êxito no cuidado aos pacientes infectados.

A análise das publicações mostrou que a ciência tem cumprido seu papel e tem oferecido à sociedade respostas rápidas, considerando a gravidade e a velocidade de disseminação da COVID-19, pois um número razoável de publicações sobre o tema dos trabalhadores e sua saúde já está disponível.

No entanto, algumas nuances sobre a questão ainda carecem de um olhar mais atento dos pesquisadores. A desigualdade observada na distribuição da produção científica pode vir a ser melhor gerenciada à medida que se acumulam experiências nos diferentes países sobre os impactos da doença entre os trabalhadores da saúde. 


\section{Contribuições dos autores}

Ribeiro AP, Oliveira GL contribuíram na concepção, levantamento dos dados, análise e interpretação de dados, escrita do texto e aprovação da versão final; Silva LS contribuiu na análise e interpretação de dados, escrita do texto e aprovação da versão final; Souza ER contribuiu na escrita do texto, revisão e aprovação da versão final. Todos os autores se responsabilizam integralmente por este trabalho e o conteúdo publicado.

\section{Referências}

1. Koh D. Occupational risks for covid-19 infection. Occup Med. 2020;70(1):3-5.

2. Brasil. Ministério da Saúde. COVID19 Painel Coronavírus [Internet]. 2020 [citado em 25 jun 2020]. Disponível em: https://covid.saude.gov.br/

3. Ferioli M, Cisternino C, Leo V, Pisani L, Palange $\mathrm{P}, \mathrm{Nava} \mathrm{S}$. Protecting healthcare workers from SARS-CoV-2 infection: practical indications. Eur Respir Rev. 2020;29(155):200068.

4. World Health Organization. Rational use of personal protective equipment for coronavirus disease (covid-19) and considerations during severe shortages. Geneva: WHO; 2020.

5. Adalja AA, Toner E, Inglesby TV. Priorities for the US health community responding to COVID-19. JAMA. 2020;323(14):1343-4.

6. Barreto ML, Barros AJD, Carvalho MS, Codeço CT, Hallal PRC, Medronho RA et al. O que é urgente e necessário para subsidiar as políticas de enfrentamento da pandemia de COVID-19 no Brasil? Rev Bras Epidemiol. 2020;23:e200032.

7. Feldman O, Meir M, Shavit D, Idelman R, Shavit I. Exposure to a surrogate measure of contamination from simulated patients by emergency department personnel wearing personal protective equipment. JAMA. 2020;323(20):2091-3.

8. Ouslander JG. Coronavirus Disease19 in geriatrics and long-term care: an update. J Am Geriatr Soc. 2020;68(5):918-21.

9. Spinazzè A, Cattaneo A, Cavallo DM. COVID-19 outbreak in Italy: protecting worker health and the response of the Italian industrial hygienists association. Ann Work Expo Health. 2020;64(6):559-64.

10. Modi PD, Nair G, Uppe A, Modi J, Tuppekar B, Gharpure AS et al. COVID-19 awareness among healthcare students and professionals in Mumbai metropolitan region: a questionnaire-based survey. Cureus. 2020;12(4):e7514.

11. Moro M, Vigezzi GP, Capraro M, Biancardi A, Nizzero P, Signorelli C et al. 2019-novel coronavirus survey: knowledge and attitudes of hospital staff of a large Italian teaching hospital. Acta Biomed. 2020;91(3-S):29-34.

12. Shi Y, Wang J, Yang Y, Wang Z, Wang G, Hashimoto $\mathrm{K}$ et al. Knowledge and attitudes of medical staff in Chinese psychiatric hospitals regarding COVID-19. Brain Behav Immun Health. 2020;4:100064.
13. Khader Y, Al Nsour M, Al-Batayneh OB, Saadeh $\mathrm{R}$, Bashier H, Alfaqih M et al. Dentists' awareness, perception, and attitude regarding COVID-19 and infection control: cross-sectional study among Jordanian dentists. JMIR Public Health Surveill. 2020;6(2):e18798.

14. Lima CKT, Carvalho PMM, Lima IAAS, Nunes JVAO, Saraiva JS, Souza RI et al. The emotional impact of Coronavirus 2019-nCoV (new Coronavirus disease). Psychiatry Res. 2020;287:112915.

15. Ho HC, Hughes T, Bozlu M, Kadığlu A, Somani BK. What do urologists need to know: diagnosis, treatment, and follow-up during COVID-19 pandemic. Turk J Urol. 2020;46(3):169-77.

16. Correia MITD, Ramos RF, Von Bahten LC. The surgeons and the COVID-19 pandemic. Rev Col Bras Cir. 2020;47(1):e20202536.

17. Xiang YT, Jin Y, Wang Y, Zhang Q, Zhang L, Cheung T. Tribute to health workers in China: A group of respectable population during the outbreak of the COVID-19. Int J Biol Sci. 2020;16(10):1739-40.

18. Heinzerling A, Stuckey MJ, Scheuer T, Xu M, Perkins KM, Resseger H et al. Transmission of COVID-19 to health care personnel during exposures to a hospitalized patient - Solano County, California, February 2020. MMWR Morb Mortal Wkly Rep. 2020;69(15):472-6.

19. Kowalski LP, Sanabria A, Ridge JA, Ng WT, de Bree R, Rinaldo A et al. COVID-19 pandemic: effects and evidence-based recommendations for otolaryngology and head and neck surgery practice. Head Neck. 2020;42(6):1259-67.

20. Keeley AJ, Evans C, Colton H, Ankcorn M, Cope A, State A et al. Roll-out of SARS-CoV-2 testing for healthcare workers at a large NHS Foundation Trust in the United Kingdom, March 2020. Euro Surveill. 2020;25(14):2000433.

21. Chu J, Yang N, Wei Y, Yue H, Zhang F, Zhao J et al. Clinical characteristics of 54 medical staff with COVID-19: a retrospective study in a single center in Wuhan, China. J Med Virol. 2020;92(7):807-13.

22. Liu M, He P, Liu HG, Wang XJ, Li FJ, Chen S et al. Clinical characteristics of 30 medical workers infected with new coronavirus pneumonia. Zhonghua Jie He Hu Xi Za Zhi. 2020;43(0): E016.

23. Xing Y, Mo P, Xiao Y, Zhao O, Zhang Y, Wang F. Post-discharge surveillance and positive 
virus detection in two medical staff recovered from coronavirus disease 2019 (COVID-19), China, January to February 2020. Euro Surveill. 2020;25(10):2000191.

24. Reusken CB, Buiting A, Bleeker-Rovers C, Diederen B, Hooiveld M, Friesema I et al. Rapid assessment of regional SARS-CoV-2 community transmission through a convenience sample of healthcare workers, the Netherlands, March 2020. Euro Surveill. 2020;25(12):2000334.

25. Lai J, Ma S, Wang Y, Cai Z, Hu J, Wei N et al. Factors associated with mental health outcomes among health care workers exposed to coronavirus disease 2019. JAMA Netw Open. 2020;3(3):e203976.

26. Lu W, Wang H, Lin Y, Li L. Psychological status of the medical workforce during the COVID-19 pandemic: a cross-sectional study. Psychiatry Res. 2020; 288:112936.

27. Wu W, Zhang Y, Wang, P, Zhang L, Wang G, Lei G et al. Psychological stress of medical staff during outbreak of COVID-19 and adjustment strategy. J Med Virol. 2020;10.1002/jmv.25914.

28. Huang JZ, Han MF, Luo TD, Ren AK, Zhou XP. Mental health survey of medical staff in a tertiary infectious disease hospital for COVID-19. Zhonghua Lao Dong Wei Sheng Zhi Ye Bing Za Zhi. 2020;38(3):192-5.

29. Xiao H, Zhang Y, Kong D, Li S, Yang N. The effects of social support on sleep quality of medical staff treating patients with coronavirus disease 2019 (COVID-19) in January and February 2020 in China. Med Sci Monit. 2020;5(26):e923549-1-e923549-8.

30. Cai H, Tu B, Ma J, Chen L, Fu L, Jiang Y, Zhuang Q. Psychological impact and coping strategies of frontline medical staff in Hunan between January and March 2020 during the outbreak of coronavirus disease 2019 (COVID19) in Hubei, China. Med Sci Monit. 2020;15(26):e924171-1-e924171-16.

31. Wu Y, Wang J, Luo C, Hu S, Lin X, Anderson AE et al. A comparison of burnout frequency among oncology physicians and nurses working on the front lines and usual wards during the COVID-19 epidemic in Wuhan, China. J Pain Symptom Manag. 2020;60(1):e60-e65.

32. Zhao Z, Gao D. Precaution of 2019 novel coronavirus infection in department of oral and maxillofacial surgery. Br J Oral Maxillofac Surg. 2020;58(3):250-3.

33. Kimmig R, Verheijen RH, Rudnicki M. Robot assisted surgery during the COVID-19 pandemic, especially for gynecological cancer: a statement of the Society of European Robotic Gynaecological Surgery (SERGS). J Gynecol Oncol. 2020;31(3):e59.

34. Xu K, Lai XQ, Liu Z. Suggestions for prevention of 2019 novel coronavirus infection in otolaryngology head and neck surgery medical staff. Zhonghua Er Bi Yan Hou Tou Jing Wai Ke Za Zhi. 2020;55(0):E001.
35. Gallasch CH, Cunha ML, Pereira LAS, Silva-Junior JS. Prevenção relacionada à exposição ocupacional do profissional de saúde no cenário de COVID-19. Rev Enferm UERJ. 2020;28:e49596.

36. Agalar C, Öztürk Engin D. Protective measures for COVID-19 for healthcare providers and laboratory personnel. Turk J Med Sci. 2020;50(SI-1):578-84.

37. Bahl P, Doolan C, Silva C, Chughtai AA, Bourouiba L, MacIntyre CR. Airborne or droplet precautions for health workers treating coronavirus disease 2019? J Infect Dis. 2020:jiaa189.

38. Jiang Q, Song S, Zhou J, Liu Y, Chen A, Bai Y et al. The prevalence, characteristics, and prevention status of skin injury caused by personal protective equipment among medical staff in fighting COVID-19: a multicenter, cross-sectional study. Adv Wound Care. 2020;9(7):357-64.

39. Luo Y, Zhong M. Standardized diagnosis and treatment of colorectal cancer during the outbreak of novel coronavirus pneumonia in Renji hospital. Zhonghua Wei Chang Wai Ke Za Zhi. 2020;23(3):E003.

40. Jamieson DJ, Steinberg JP, Martinello, RA, Perl T, Rasmussen SA. Obstetricians on the coronavirus disease 2019 (COVID-19) front lines and the confusing world of personal protective equipment. Obstet Gynecol. 2020;135(6):1257-63.

41. Massey PA, McClary K, Zhang AS, Savoie FH, Barton RS. Orthopaedic surgical selection and inpatient paradigms during the coronavirus COVID-19 pandemic. J Am Acad Orthop Surg. 2020;10.5435/JAAOS-D-20-00360.

42. Tan YT, Wang JW, Zhao K, Han L, Zhang HQ, Niu HQ et al. Preliminary recommendations for surgical practice of neurosurgery department in the central epidemic area of 2019 coronavirus infection. Curr Med Sci. 2020;40(2):281-4.

43. Zhang MC, Xie HT, Xu KK, Cao Y. Suggestions for disinfection of ophthalmic examination equipment and protection of ophthalmologist against 2019 novel coronavirus infection. Zhonghua Yan Ke Za Zhi. 2020;56(0):E001.

44. Li W, Huang J, Guo X, Zhao J, Mandell MS. Anesthesia management and perioperative infection control in patients with the novel coronavirus. J Cardiothorac Vasc Anesth. 2020;S1053-0770(20)30284-6 .

45. Ding J, Fu H, Liu Y, Gao J, Li Z, Zhao X et al. Prevention and control measures in the radiology department for COVID-19. Eur Radiol. 2020;30:3603-8.

46. Setiati S, Azwar MK. COVID-19 and Indonesia. Acta Med Indones. 2020;52(1): 84-9.

47. Gan WH, Lim JW, Koh D. Preventing intrahospital infection and transmission of COVID-19 in healthcare workers. Saf Health Work. 2020;11(2):241-3. 
48. Peng X, Xu X, Li Y, Cheng L, Zhou X, Ren B. Transmission routes of 2019-nCoV and controls in dental practice. Int J Oral Sci. 2020;12(1).

49. Ma SY, Yuan ZQ, Peng YZ, Luo QZ, Song HP, Xiang $\mathrm{F}$ et al. Recommendations for the regulation of medical practices of burn treatment during the outbreak of the coronavirus disease 2019. Zhonghua Shao Shang Za Zhi. 2020;36(6):465-9.

50. Huang L, Lin G, Tang L, Yu L, Zhou Z. Special attention to nurses' protection during the COVID-19 epidemic. Crit Care. 2020;24(1):120.

51. Johns Hopkins University \& Medicine. Coronavirus Resource Center [Internet]. 2020 [citado em 25 jun 2020]. Disponível em: https:// coronavirus.jhu.edu/map.html

52. Balla M, Merugu GP, Patel M, Koduri NM, Gayam V, Adapa S et al. COVID-19, modern pandemic: a systematic review from front-line health care providers' perspective. J Clin Med Res. 2020;12(4):215-29.

53. Jackson Filho JM, Assunção AA, Algranti E, Garcia EG, Saito CA, Maeno M. A saúde do trabalhador e o enfrentamento da COVID-19. Rev Bras Saúde Ocup. 2020;45:e14.

54. Agência Brasil. No Brasil, 31790 profissionais de saúde contraíram COVID-19 [Internet]. 2020. [citado em 22 maio 2020]. Disponível em: https:// agenciabrasil.ebc.com.br/saude/noticia/2020-05/ no-brasil-31790-profissionais-de-saudecontrairam-covid-19

55. Observatório Enfermagem [Internet]. 2020 [citado em 22 maio 2020]. Disponível em: http:// observatoriodaenfermagem.cofen.gov.br/

56. World Health Organization. Coronavirus disease (COVID-19) outbreak: rights, roles and responsibilities of health workers, including key considerations for occupational safety and health. Interim guidance, 2020 [Internet]. 2020 [citado em 22 jun 2020]. Disponível em: https://www.who. $\mathrm{int} /$ publications/i/item/coronavirus-disease-(covid19)-outbreak-rights-roles-and-responsibilities-of- health-workers-including-key-considerations-foroccupational-safety-and-health

57. Zhu W, Huang X, Zhao H, Jiang X. A COVID-19 patient who underwent endonasal endoscopic pituitary adenoma resection: a case report. Neurosurgery. 2020;87(2):E140-E146.

58. Jones RM, Bleasdale SC, Maita D, Brosseau LM, CDC Prevention Epicenters Program. A systematic risk-based strategy to select personal protective equipment for infectious diseases. Am J Infect Control. 2020;48(1):46-51.

59. Tang LY, Wang J. Anesthesia and COVID-19: What we should know and what we should do. Semin Cardiothorac Vasc Anesth. 2020;24(2):127-37.

60. Malhotra N, Gupta N, Ish S, Ish P. COVID-19 in intensive care. Some necessary steps for health care workers. Monaldi Arch Chest Dis. 2020;90(1):161-2. https://doi.org/10.4081/ monaldi.2020.1284

61. Mandrola J. CoViD-19 e dispositivi di protezione individuale: qualcuno di noi morirà per la loro carenza. Recenti Prog Med. 2020;111(4):183.

62. Li Z, Ge J, Yang M, Feng J, Qiao M, Jiang R et al. Vicarious traumatization in the general public, members, and non-members of medical teams aiding in COVID-19 control. Brain Behav Immun. 2020;S0889-1591(20)30309-3.

63. Hua CG, Liu ZQ, Wang Q, Yang Z, Xu QH, Zhang J. Strategy of dental clinics to cope with the epidemic period of infectious diseases based on the experience of corona virus disease outbreak. Hua Xi Kou Qiang Yi Xue Za Zhi. 2020;38(2):117-21.

64. Liu Y, Jin GF, Wang JM, Xia YK, Shen HB, Wang CQ et al. Thoughts on the Reform of Preventive Medicine Education in the Context of New Medicine. Zhonghua Yu Fang Yi Xue Za Zhi. 2020;54(0):E030.

65. Li H, Zheng S, Liu F, Liu W, Zhao R. Fighting against COVID-19: innovative strategies for clinical pharmacists. Res Social Adm Pharm 2020;S15517411(20)30328-4. 